\title{
MMP19 is upregulated during melanoma progression and increases invasion of melanoma cells
}

Matthias Müller ${ }^{1,8}$, Inken M Beck ${ }^{2,3,8}$, Judith Gadesmann ${ }^{4}$, Nadine Karschuk ${ }^{1}$, Annette Paschen $^{5}$, Ehrhard Proksch ${ }^{6}$, Valentin Djonov ${ }^{7}$, Karina Reiss ${ }^{1,6}$ and Radislav Sedlacek ${ }^{2,3}$

${ }^{1}$ Department of Biochemistry, University of Kiel, Kiel, Germany; ${ }^{2}$ Institute of Biotechnology, Prague, Czech Republic; ${ }^{3}$ Institute of Molecular Genetics, Prague, Czech Republic; ${ }^{4}$ Department of Dermatology, Hospital of the German Armed Forces, Ulm, Germany; ${ }^{5}$ Clinical Cooperation Unit Dermato-Oncology, German Cancer Research Center Heidelberg, University Medical Center Mannheim, Mannheim, Germany; ${ }^{6}$ Department of Dermatology, University of Kiel, Kiel, Germany; and ${ }^{7}$ Department of Medicine, Gross Anatomy and Vascular Biology, University of Fribourg, Fribourg, Switzerland

During the progression of cutaneous melanomas, matrix metalloproteinases (MMPs) facilitate the tumour cells to traverse the basement membrane and invade the dermis. In this study, we analysed the expression of MMP19 in the course of melanoma progression. Although MMP19 was absent in melanocytes and melanoma cells of early stages of melanoma development, its expression was strongly upregulated in the neighbouring keratinocytes that may facilitate the vertical outgrowth of melanoma cells. In contrast to early stages, MMP19 was upregulated during the vertical growth phase of melanoma and in metastases. The upregulation of MMP19 in melanoma of Clark levels IV and V correlates with that of MMP2 and also simultaneously with ceased expression of E-cadherin. To reveal whether MMP19 facilitates the invasion of melanomas, we examined adhesion and migratory capacity of selected melanoma cell lines. Melanoma cell lines with low expression of MMP19 exhibited increased adhesion to various substrates and lower migration in comparison with the cell line with higher expression of MMP19. Moreover, ectopic expression of MMP19 could restore the migratory capacity of melanoma cells with low endogenous level of MMP19. These results suggest that the increase of MMP19 expression hallmarks the progression of cutaneous melanoma and might augment melanoma growth by promoting the invasion of tumour cells.

Modern Pathology (2010) 23, 511-521; doi:10.1038/modpathol.2009.183; published online 22 January 2010

Keywords: melanoma; invasion; skin; matrix metalloproteinase

Melanomas are malignant tumours characterized by a highly invasive and metastatic potential. During melanoma progression, tumour cells cross the basement membrane and invade the dermal compartment. Degradation of the basement membrane and surrounding extracellular matrix belongs to the critical steps of melanoma progression. ${ }^{1-3}$ This progression of cutaneous melanoma is usually

Correspondence: Dr R Sedlacek, PhD, Department of Transgenic Models of Diseases, Institute of Molecular Genetics AS CR v.v.i., Videnska 1083, 14220 Prague 4, Czech Republic.

E-mail: radislav.sedlacek@img.cas.cz

${ }^{8}$ These authors contributed equally to this work.

Received 11 June 2009; revised 25 November 2009; accepted 7

December 2009; published online 22 January 2010 divided into four stages: (a) tumours in situ, (b) radial growth phase melanomas, (c) vertical growth phase melanomas, and (d) metastatic melanomas. Although radial growth phase melanomas exhibit very limited dermal infiltration and no metastatic capacity vertical growth phase melanomas are strongly invasive and posses high potential to build metastasis. ${ }^{4}$ Clinically, developmental stages of melanoma are classified by their level of invasiveness (Clark levels (CLs) $\mathrm{I}-\mathrm{VI})^{5}$ and the tumour thickness ${ }^{6}$ that mainly serve as prognostic factors.

Tumour growth, its invasion, and metastasis depend on proliferative, adhesive, and proteolytic mechanisms. The proteolysis that leads to destruction of original tissue and rebuilding the tumour surroundings enable the penetration of neoplastic 
cells. The main degrading enzymes are members of the matrix metalloproteinase (MMP) family, which are able to degrade large number of components of the extracellular matrix. ${ }^{1,7,8}$ Under physiological conditions, most cells exhibit no or low expression of MMPs and their activity is in equilibrium with their inhibitors. This balance is, however, deregulated in various human cancer types.

Expression of MMP2, MT1-MMP, and MMP1 correlates with melanoma progression..$^{9-14}$ Thereby, MT1-MMP directly degrades components of the basal membrane and also contributes to the activation of MMP2. Strong upregulation of MMP1 and MMP2 is clearly connected to melanoma cell invasion. ${ }^{15}$ MMP1 is expressed in melanoma cells invading the dermis in which it is responsible not only for degradation of collagen type I but also for activation of TGF- $\beta$ by degrading latency-associated peptide. ${ }^{12,16}$ MMP13 seems to be related to an aggressive phenotype of melanoma. ${ }^{17,18}$ In contrast to MMP2, MMP9 and MMP21 seem to have a more essential function in early events of melanoma formation and are downregulated in invasive stages. ${ }^{18,19}$

In parallel to extracellular matrix degradation, adhesive characteristic of tumour cells during melanoma progression is changing. In melanocytes of healthy epidermis, E-cadherin serves as important cell-cell adhesion molecule responsible for homeostasis in the skin. During melanoma development, E-cadherin is downregulated, whereas $\mathrm{N}$-cadherin facilitates interactions of tumour cells with stroma cells. ${ }^{20,21}$

In contrast to other MMPs in the skin, MMP19 is constitutively expressed in basal keratinocytes of healthy epidermis. ${ }^{22}$ Dysregulated MMP19 expression in the epidermis was described in different skin diseases associated with hyperproliferation such as psoriasis, tinea, and eczema. ${ }^{22}$ Upregulation of MMP19 was also observed in epithelial cells within neoplastic differentiation of the mammary gland, in squamous cell carcinoma (SCC), ${ }^{23-25}$ and in macrophages during inflammation. ${ }^{26-28}$ MMP19 is able to cleave several components of the extracellular matrix such as collagen IV, nidogen, tenascin C, laminin $5 \gamma_{2}$, fibronectin, cartilage oligomeric protein, aggrecan, and secreted insulin-like growth factor binding protein 3 , thus modulating IGF signaling pathway. ${ }^{25,29-32}$

In this study, we evaluated the expression of MMP19 in cutaneous melanoma at different stages of progression using immunohistochemistry. We found that the expression of MMP19 increases during the melanoma progression and that MMP19 activity facilitates the migration of tumour cells.

\section{Materials and methods}

\section{Tissue Samples and Classification}

Paraffin-embedded tissue samples from melanoma patients and sections of healthy human skin were obtained from the Department of Dermatology, University of Kiel, with approval of the Regional Board of the Medical Ethics Commission and with written consent of the patients. Tissue samples, classified in different CLs as marker of invasiveness from 66 individuals, were analysed (see Table 1). Images were captured using microscope Axioskop 2 (Zeiss, Jena, Germany).

\section{Immunohistochemistry}

Tissue specimens embedded in paraffin were cut and deparaffinized. Endogenous peroxidase activity was blocked by $\mathrm{H}_{2} \mathrm{O}_{2}$. Antigen retrieval was performed by treating sections with $10 \mathrm{mM}$ citric acid, $\mathrm{pH} 6.0$ at $90^{\circ} \mathrm{C}$ for $30 \mathrm{~min}$ and with $0.05 \%$ trypsin (Roth, Karlsruhe, Germany) in TBS for $10 \mathrm{~min}$ at $37^{\circ} \mathrm{C}$. To detect MMP19, the monoclonal anti-MMP19 antibody CK8/4 was used as described. ${ }^{33}$ For detection of melanoma cells, monoclonal anti-Melan-A antibodies (DakoCytomation, Glostrup, Denmark) were used. Sections were also stained with monoclonal antibodies against MMP2 and MMP9 (both NeoMarkers, Union City, CA, USA). Bound antibodies were detected using biotinylated species-specific antibodies (Vectastain Elite kit, Vector, Burlingham, CA, USA). The reaction was visualized with 3-amino-9ethylcarbazole substrate (AEC; Vector).

Table 1 Evaluation of immunohistochemical staining of MMP19 during melanoma progression

\begin{tabular}{|c|c|c|c|c|c|c|}
\hline & CLI in situ & CLII & CLIII & $C L I V$ & $C L V$ & In-transit metastasis \\
\hline Number of samples & 9 & 22 & 13 & 11 & 5 & 6 \\
\hline MMP19—positive cells (s.d.) & $0.1(0.31)$ & $0.84(0.66)$ & $1.27(0.89)$ & $2.64(0.48)$ & $3(0.65)$ & $3(0)$ \\
\hline MMP19_staining intensity (s.d.) & $0.1(0.31)$ & $0.95(0.77)$ & $1.1(0.27)$ & $1.82(0.38)$ & $2(0.40)$ & $2.3(0.75)$ \\
\hline $\begin{array}{l}\text { MMP19 in keratinocytes } \\
\text { (suprabasal staining) }\end{array}$ & $11 \%(1$ of 9$)$ & $77 \%(17$ of 22$)$ & $85 \%$ (11 of 13$)$ & $100 \%(11$ of 11$)$ & $100 \%(5$ of 5$)$ & \\
\hline MMP19 (staining of fibroblasts) & 0 & $62 \%(13$ of 21$)$ & $46 \%(5$ of 11$)$ & $91 \%(10$ of 11$)$ & $100 \%(5$ of 5$)$ & $100 \%(6$ of 6$)$ \\
\hline
\end{tabular}




\section{Semi-quantitative Evaluation of Staining}

Staining was assessed independently by two investigators at a discussion microscope. Staining intensity (SI) was graded semi-quantitatively by numbers as follows: 0, not detected; 1, minimal; 2 , moderate; 3 , strong. Both the SI and the number of positive cells (PC) were evaluated. The PC number was defined as follows: 0 , no staining; 1 , rare $(<25 \%) ; 2$, moderate $(25-50 \%)$; and 3 , frequent $(>50 \%)$. In consequence, 10 categories, each described by two numbers, were established: $(0,0)$; $(1,1) ;(1,2) ;(1,3) ;(2,1) ;(2,2) ;(2,3) ;(3,1) ;(3,2)$, and $(3,3)$, whereby the first number represents the grade of immunoreactivity against MMP19 antibody, and the second defines the amount of total MMP19-PC. Staining of MMP19 above the basal layer of the epidermis was scored with 1 (positive staining) and missing signal for MMP19 was scored by 0 (negative). Dermal fibroblasts were also screened for MMP19 staining $(0=$ not detected, $1=$ positive staining). For each parameter, at least four fields per tissue sample were evaluated at a 400-fold magnification as described earlier. ${ }^{15,34}$

\section{Cell Lines and Culture Conditions}

The human melanoma cell lines UKRV-Mel-15a, UKRV-Mel-21a, MaMel-45a, and MaMel-54a (referred as Mel-15a, Mel-21a, Mel-45a, and Mel-54a) were cultured in RPMI1640 (PAA, Linz, Austria) under conditions as described earlier. ${ }^{35}$ For adhesion and transmigration assays, cells were starved in medium without FCS for $24 \mathrm{~h}$.

\section{Western Blot Analysis and Gelatinolytic Zymogram}

For western blotting, lysates of melanoma cell lines were obtained as described. ${ }^{32}$ Detection of protein expression was performed with antibodies against MMP19 (AffinityBioReagents, Golden, CO, USA). Analysis of MMP2 and MMP9 expression by gelatinolytic zymography was carried out from cell supernatants normalized to cell numbers as described earlier. ${ }^{36}$

\section{Cell Proliferation, Adhesion, and Transmigration Assays}

Proliferation was assessed by ${ }^{3} \mathrm{H}$-thymidine incorporation as described. ${ }^{32,36}$ For adhesion assays, 96well plates were coated with poly-D-lysine (Roche, Indianapolis, NI, USA), fibronectin (Invitrogen, Carlsbad, CA, USA), collagen type I, or type IV matrices (both BD Biosciences, San Jose, CA, USA) in concentrations of $5 \mu \mathrm{g} / \mathrm{cm}^{2}$. Cells stained with calcein (Molecular Probes, Eugene, OR, USA) were seeded at 15,000 cells/well. Fluorescence of adherent and non-adherent cells was measured after $2 \mathrm{~h}$ at $530 \mathrm{~nm}$ using a microplate reader (Lambda Fluoro
320, MWG Biotech, Germany). For transmigration, Nunclon Cell Culture Inserts (Nunc, Wiesbaden, Germany) were used. Inserts were coated with fibrin matrix, collagen type I, or type IV $\left(10 \mu \mathrm{g} / \mathrm{cm}^{2}\right)$. Calcein-stained cells at 150,000 cells/well were seeded in the upper chamber containing serum-free medium, whereas the lower chamber was filled with FCS-containing medium. Transmigrated cells were measured after 10 and $20 \mathrm{~h}$ by detecting fluorescence in the lower chamber and in total. Values are given in percent transmigrated cells. Assays were performed two times in quadruplicates for each sample.

\section{Transfection of Melanoma Cells}

Melanoma cells Mel-21a were transiently transfected with human MMP19 constructs wild type (WT) and EA (inactive MMP19) cloned into pIRES vector as described. ${ }^{32}$ Transfection was performed using Fugene6 (Roche) and empty vector was used as a control. In cell lysates, the efficiency of transfection was determined by western blotting against MMP19.

\section{Statistical Analysis}

Statistical analysis was carried out using ANOVA and the Student's $t$-test (SigmaStat for Windows, Analytical Software Program). The results are presented as mean \pm standard error of the mean with significant values at $P<0.05$.

\section{Results}

MMP19 Expression is Absent in Early Melanoma but Upregulated in Adjacent Keratinocytes

In healthy human skin, immunostaining for MMP19 was generally restricted to the undifferentiated population of keratinocytes within the basal epidermal layer, whereas no expression of MMP19 was observed in melanocytes and suprabasal-differentiated keratinocytes. As earlier described, the expression pattern of MMP19 corresponded to that of cytokeratin 14, a marker of undifferentiated basal keratinocytes. ${ }^{22,32}$ To distinguish between tumour and non-tumour cells, melanoma cell marker Melan-A was used. In melanoma in situ (Figure 1; Table 1) Melan-A-PC (Figure 1b) did not show obvious immunoreactivity for MMP19 (Figure 1a). Hence, MMP19 staining and the SI in Melan-A-PC were defined as negative $(0,0)$ in all samples studied. Upregulation of MMP19 in suprabasal keratinocytes was found only in one out of nine samples. Furthermore, dermal fibroblasts did not show any elevated expression of MMP19 (Figure 1; Table 1). 

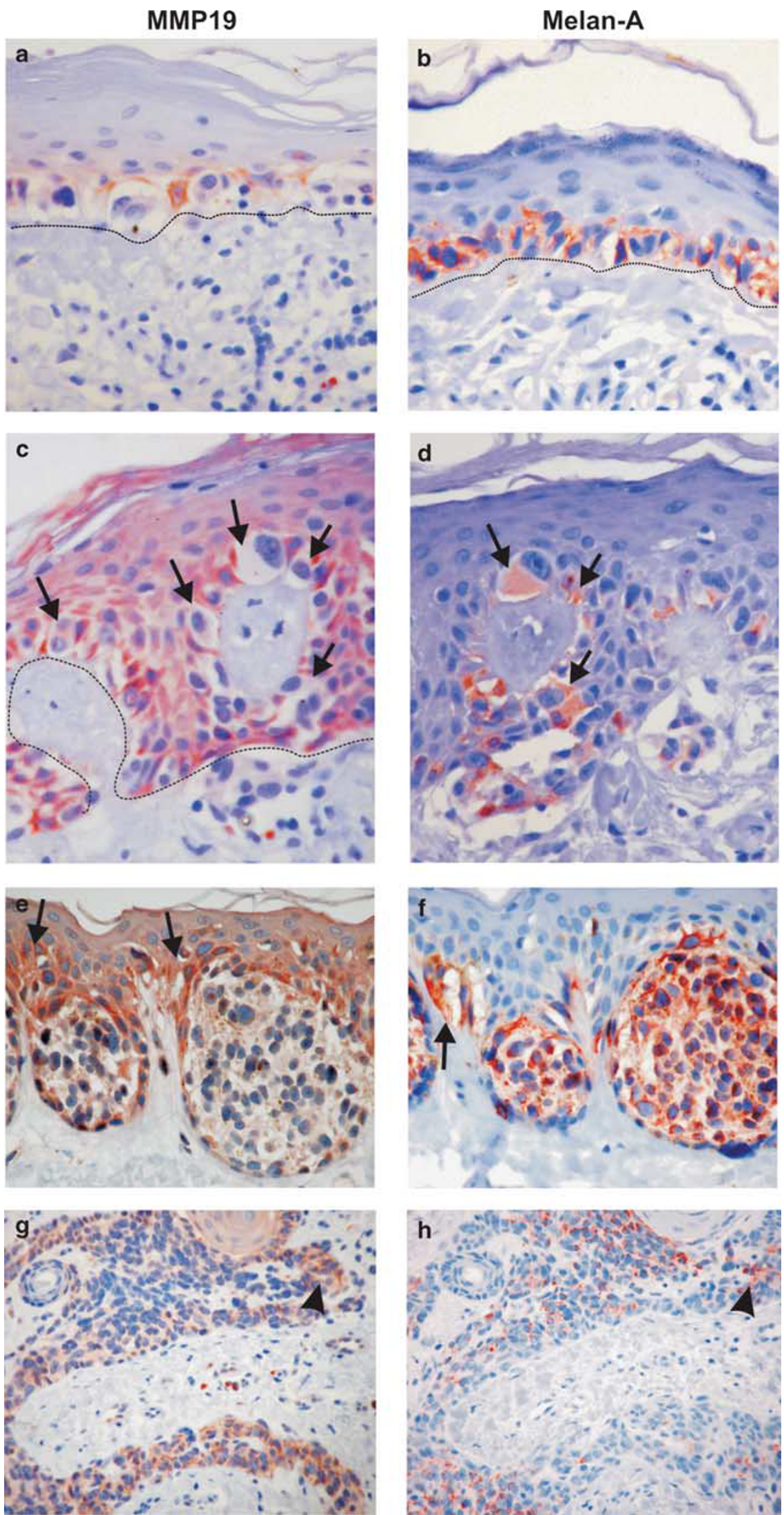
Upregulation of MMP19 in Low Invasive Stages Clark II and III

Both Clark II and Clark III melanomas showed week but sustained staining for MMP19 in tumour cells. Clark II melanoma cells characterized by the expression of Melan-A were largely negative for MMP19; however, 17 out of $22(77 \%)$ biopsies showed apparent upregulation of MMP19 in suprabasal keratinocytes (Figure 1c). The mean staining score and the standard error of mean for the number of MMP19-positive melanoma cells and the intensity of immunostaining (SI) were assigned as 0.84 $( \pm 0.66)$ and $0.95( \pm 0.77)$, respectively. The expression of MMP19 in dermal fibroblasts was observed in 13 out of $21(62 \%)$ biopsies (summarized in Table 1).

In melanoma with intermediate level of invasiveness (Clark III), the amount of MMP19-PC $(1.27 \pm 0.89)$ as well as the SI $(1.1 \pm 0.27)$ seemed to be higher than in Clark II melanoma, (Figure 1c) although the expression of MMP19 exhibited no statistical significance. In correlation with earlier investigations, ${ }^{37}$ Clark III melanoma showed positive staining for MMP9 and to lesser extent for MMP2 (not shown). Fibroblasts and suprabasal keratinocytes in Clark III biopsies also showed MMP19 expression in 85\% (11 of 13) and 46\% (5 of 11), respectively (Table 1). Moreover, keratinocytes adjacent to melanoma cells exhibited very intensive MMP19 staining in comparison to more distal keratinocytes in suprabasal layers (Figure 1e and f). To evaluate apoptotic processes within Clark III tumours, TUNEL staining was performed. Only few nuclei of melanoma cells showed positive staining (7.6 out of 100; not shown).

\section{Advanced Malignant Melanomas and Melanomas with Early Metastasis Show High Expression of MMP19}

In advanced malignant melanomas, the number of MMP19-PC and the SI for MMP19 showed significantly higher values in comparison with lower invasive stages (Figure 1g; Table 1). The number of MMP19-PC in Clark IV (Figure 1g) or Clark V (Figure 2a) tumours was increased twofold com- pared with Clark III melanomas $(2.64 \pm 0.48$ in Clark IV, $3 \pm 0.65$ in Clark V vs $1.27 \pm 0.89$ in Clark III). In addition, the SI of MMP19 showed a significant increased value of $1.82 \pm 0.38(P<0.05)$ in Clark IV and $2 \pm 0.40$ in Clark V melanoma vs $1.1 \pm 0.27$ in Clark III. In Clark IV melanomas, 10 out of 11 (91\%) biopsies showed an upregulation of MMP19 in dermal fibroblasts and all biopsies revealed increased MMP19 staining in suprabasal keratinocytes. In Clark V melanomas (Figure 3a), all biopsies exhibited staining for MMP19 in suprabasal keratinocytes and dermal fibroblasts.

The staining of MMP19 in Clark IV and Clark V melanomas correlated positively with the increased expression of MMP2 (Figure 2b), whereas MMP9 disappeared in these stages (Figure 2c). Thus, the expression of MMP19 seemed to be similar with that of $\mathrm{MMP}^{15}$ and dissimilar from MMP9 that is downregulated during vertical growth, although increased immunoreactivity in vascular endothelial cells remains (Figure 2c). In Clark IV melanomas, only a small number of nuclei of tumour cells showed TUNEL staining and were considered to be apoptotic (6.8 of 100) (not shown). Primary metastases of malignant melanomas showed strong expression of MMP19 (Figure 2d) . The number of PC $(3 \pm 0)$ and the SI were slightly higher than in Clark V stages $(2.3 \pm 0.75)$.

\section{Stromal Cells Close to Melanoma Express MMP19}

Fibroblasts located near to tumour cells showed weak but significantly increased $(P<0.05)$, immunoreactivity for MMP19 during melanoma progression starting at Clark II melanoma, whereas no MMP19 could be observed in stromal fibroblast in melanoma in situ (Clark I). A number of inflammatory cells infiltrating the tumour stroma expressed MMP19 independently of the melanoma stage (Figure 3a). Tumour surrounding fibroblasts showed abundant expression of MMP19 (Figure 3b, Clark III). MMP19-positive endothelial cells of the dermis or the hypodermal fatty tissue are only found in Clark V melanomas and primary metastases, in which adipose tissue and tumour cells come close to each other (Figure 3c and d).

Figure 1 MMP19 is upregulated in tumour cells in advanced melanoma. Immunohistochemical staining of human epidermis against MMP19 (a, c, e, and g) and Melan-A (b, d, f, and h) showing melanoma in situ (a and b), Clark II (c and d), Clark III melanoma (e and f), and Clark IV (g and $\mathbf{h}$ ) melanomas. In melanoma in situ, Melan-A-PC (b) as well as dermal fibroblasts and suprabasal keratinocytes do not show immunoreactivity for MMP19 (a). The melanoma cells have not traversed the basal membrane (dotted line, a and b). Keratinocytes in early stages of melanoma (Clark II) show an upregulation of MMP19 expression in suprabasal layers, whereas MMP19 is not expressed in melanocytes and cells of melanocytic origin (c and d). Large pale cells (arrows), interspersed between MMP19-positive keratinocytes are negative for MMP19 (c) and represent highly polymorph melanoma cells confirmed by Melan-A staining (d, arrows). Position of the basal membrane is indicated (lines in $\mathbf{a}$, b, and c). In Clark III melanomas, Melan-A-positive tumour cells can be found at the basis of rete ridges (arrow, f), from where they start to infiltrate the papillary dermis. The malignant cells themselves show moderate MMP19 expression (e), whereas neighbouring keratinocytes in the Stratum basale and S. spinosum exhibit augmented expression of MMP19 (arrows, e). In Clark IV melanomas, staining for MMP19 (g) and Melan-A (h) showed similar expression pattern. During the outgrowth of melanoma nests into the dermal compartment, most of the tumour cells express MMP19 (arrowheads, comparison in $\mathbf{g}$ and h) (magnification: $\mathbf{a}-\mathbf{h}, \times 200)$. 


\section{MMP19}

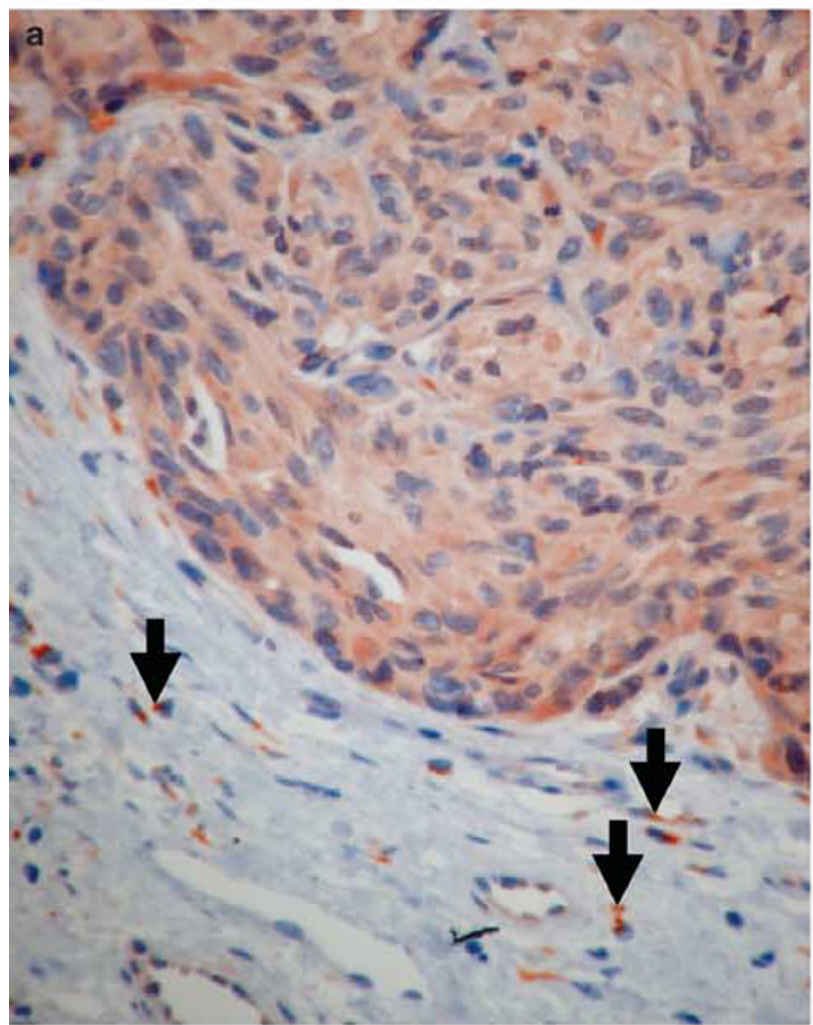

MMP9

"

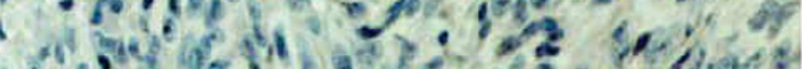

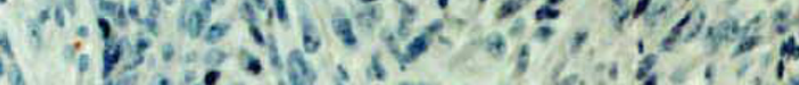

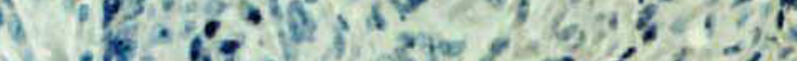

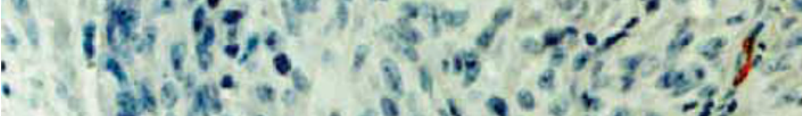

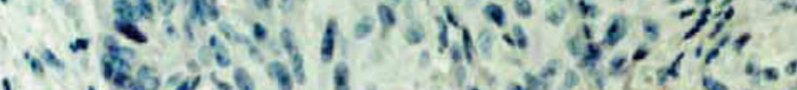

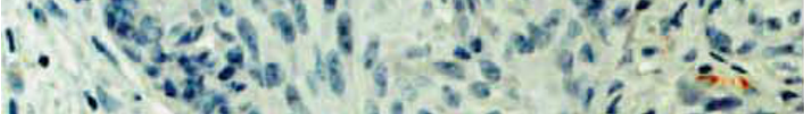
1.

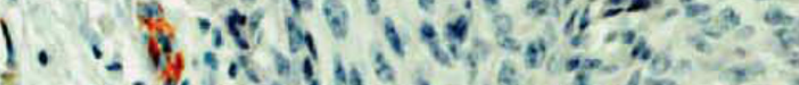

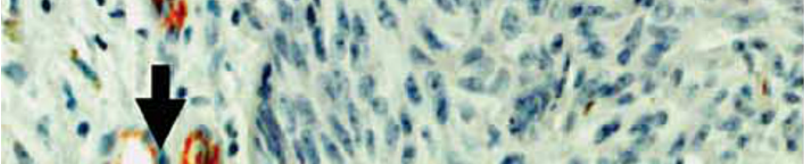
4.

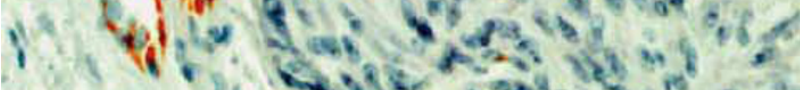

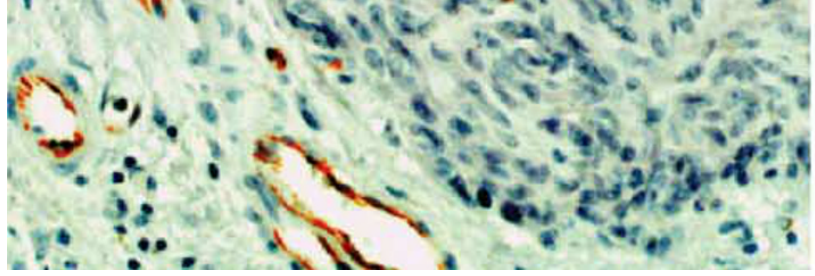

\section{MMP2}

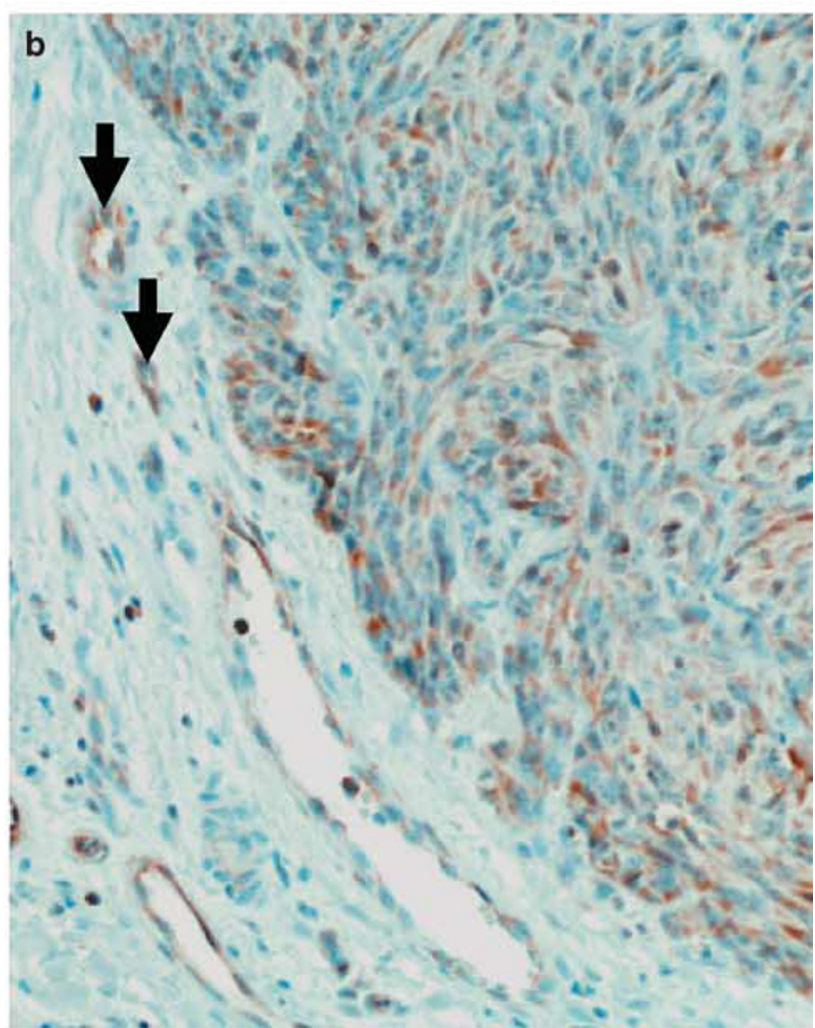

MMP19

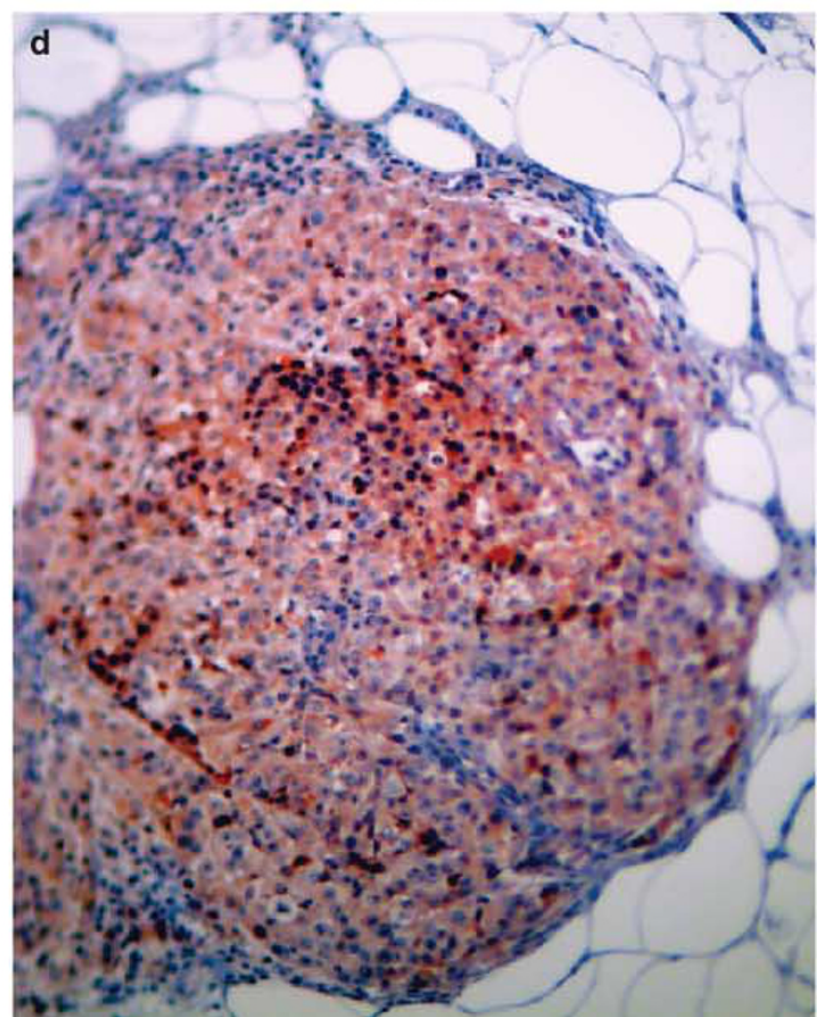




\section{Expression of MMP19 in Melanoma Cell Lines Correlates with Increased Invasion}

Experiments with human melanoma cell lines were performed to reveal whether MMP19 could facilitate invasion of tumour cells. Four cell lines, Mel-45a, Mel-54a, Mel-15a, and Mel-21a, generated from melanoma metastasis were analysed for MMP19, MMP2, and MMP9 expression (Figure 4). Cell line Mel-15a showed highest expression of MMP19 (Figure 4a). Expression of MMP2 and MMP9 was assessed by gelatinolytic zymogram that revealed no obvious alterations in their expression (Figure 4b).
To estimate the invasive capacity of melanoma cell lines, proliferation, adhesion, and transmigration were analysed. Mel-15a cells exhibited significantly reduced adhesion to fibronectin, poly-D-lysine, type I, and type IV collagen (Figure 4c); however, also highest transmigration rate through fibrin, type I, and type IV collagen matrices (Figure 5a). Mel-45a, Mel-54a, and Mel-21a cell lines showed comparable adhesion and transmigration levels. All melanoma cell lines showed a comparable proliferation rate (Figure $5 b$ ).

To find out whether MMP19 directly impacts migratory behaviour of melanoma cells, the cell line
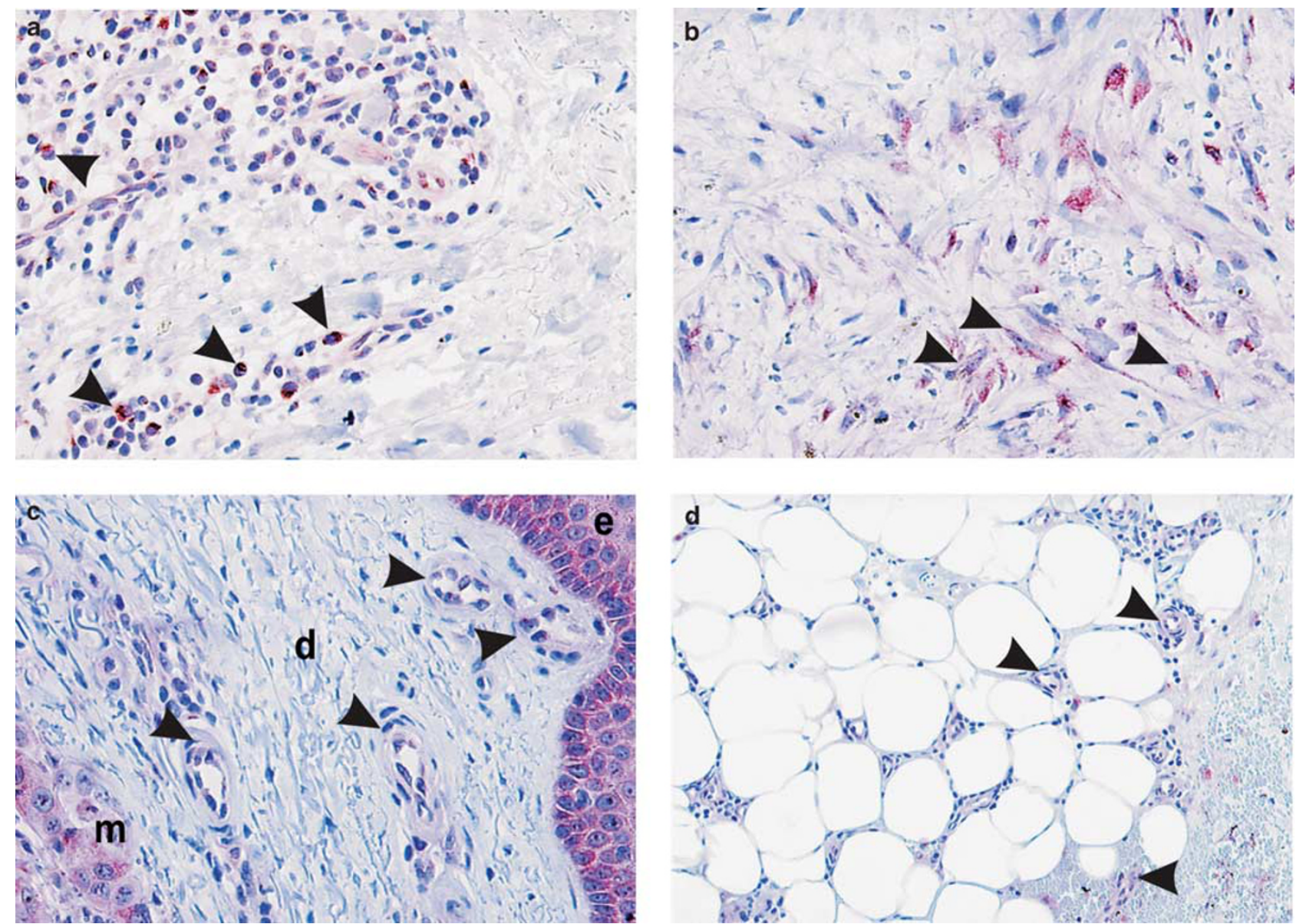

Figure 3 Different cell types of the tumour stroma are positive for MMP19. Melanoma of different invasion levels were analysed for MMP19 expression pattern in tumour surroundings (a and b, Clark III; c, Clark IV; d, Clark V). Inflammatory cells infiltrating the tumour stroma show MMP19 expression (arrowheads, a). Fibroblasts (arrowheads in b) and endothelial cells (c and d) in the neighbourhood of melanoma (m) exhibit increased MMP19 expression during melanoma progression. MMP19-positive endothelial cells of the hypodermal fatty tissue are only found in Clark V melanomas (d) and in metastasis, in which adipocytes and tumour cells come close to each other (d, dermis; e, epidermis; m, melanoma) (magnification: a-d: $\times 200$ ).

Figure 2 Tumour cells in highly invasive melanomas and metastases express MMP19. Immunohistochemical staining of MMP19 (a and e), MMP2 (b), and MMP9 (c) in invasive melanomas (CL V, a-c) and a metastase (d). In Clark V melanomas, the majority of tumour cells are positive for MMP19 (a). Fibroblasts of the surrounding stroma express MMP19 (arrows, a). MMP2 is abundantly expressed in Clark V melanoma (I) as well as in adjacent endothelial cells and stromal fibroblasts (arrows, b). MMP9 is not apparent in Clark V melanoma cells, but present in vascular endothelial cells (arrow, c). Staining against MMP19 (e) in metastases reveal highly PC (magnification: a-d, $\times 200$ ). 

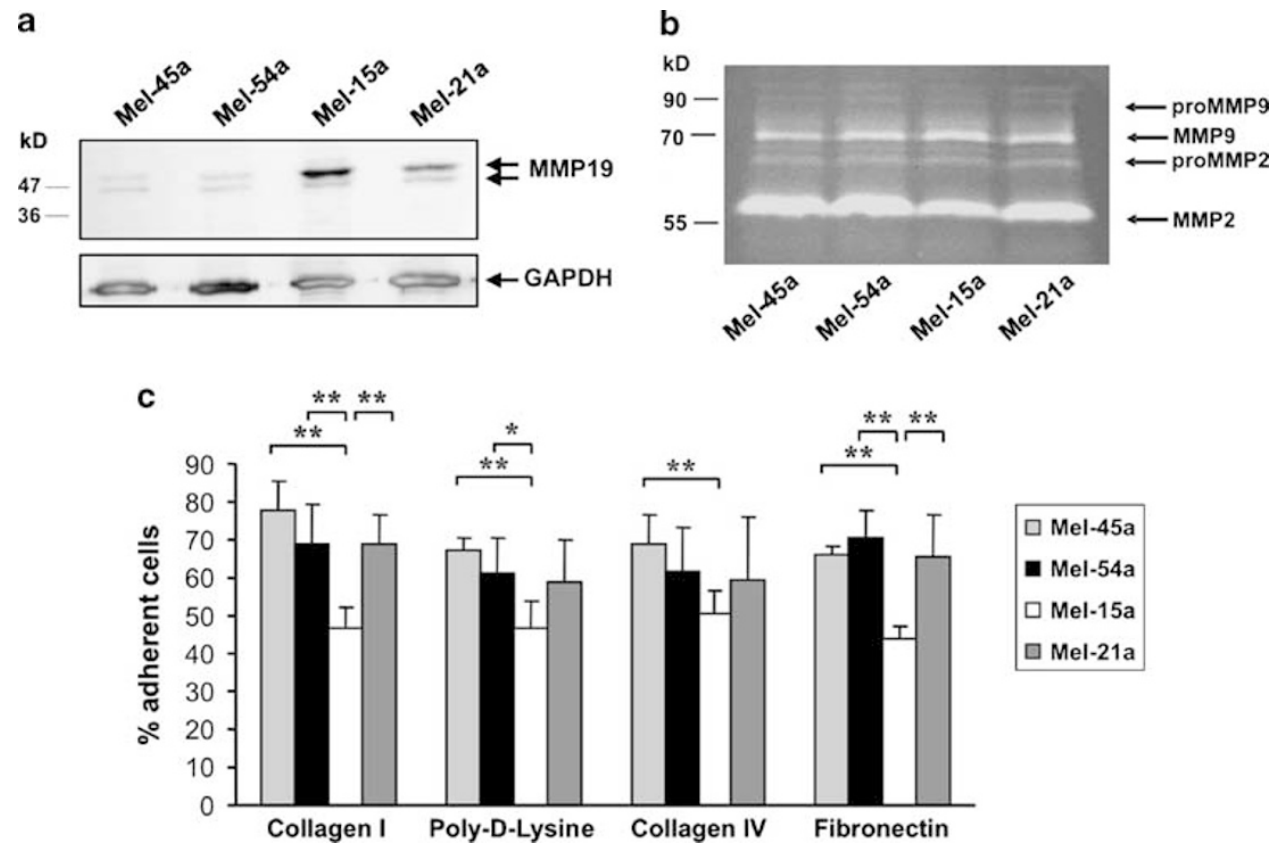

Figure 4 MMP19 in human melanoma cell lines Mel-45a, Mel-54a, Mel-15a, and Mel-21a. Expression of MMP19 (a, upper panel) was analysed by Western blotting. Unprocessed $(57 \mathrm{kDa})$ and processed forms of MMP19 are detected (arrows). GAPDH, loading control (a, lower panel). Expression of gelatinases MMP2 and MMP9 was analysed by gelatinolytic zymography (b). Adhesiveness to extracellular matrix components, ie type I and IV collagens, poly-D-lysine, and fibronectin (c).

Mel-21a exhibiting weaker expression of MMP19 was transiently transfected with active (WT) or inactive (EA) form of MMP19 (Figure 5c and d). Mel-21a ectopically expressing MMP19-WT gained higher transmigratory capacity through collagen type IV than cells expressing MMP19-EA or the control cells. Thus, active MMP19 that might be upregulated in melanoma cells, especially in the late invasion stages and during metastasis, could have the capacity to facilitate tumour cell invasion.

\section{Discussion}

The degradation of basal membrane and extracellular matrix components correlates with the invasiveness of a tumour, and many authors have already showed the importance of MMPs in this process. ${ }^{38-41}$ In this study, the expression of MMP19 in human melanoma was evaluated. Under physiological circumstances, MMP19 is expressed in undifferentiated keratinocytes but is not found in melanocytes or in early stage melanomas. It is upregulated during the later stages of tumour invasion and in metastatic cells. MMP19 shows a similar pattern to MMP2 that is upregulated in advanced invasive tumours. ${ }^{14,15,19,37,42-45}$ Schaumburg-Lever and coworkers described colocalization of MMP2, type IV collagen, and laminin at the basement membrane of melanoma. As MMP19 cleaves type IV collagen, ${ }^{32}$ it could together with other proteases change interactions between melanoma cells and their stroma and, thus, modulates the behaviour of invading tumour cells. Another matrix protein that might be efficiently cleaved by MMP19 is laminin $5 \gamma 2$ chain. ${ }^{25}$ In SCC MMP19 is colocalized with laminin 5 in the invading front of the tumour. ${ }^{25}$ In vitro studies showed that active MMP19-dependent processing of $\gamma 2$ chain leads to increased cell migratory capacity. Although the expression pattern of MMP19 in melanoma differs from that of SCC, MMP19 may also participate in melanoma progression because of processing of $\gamma 2$ chain and other basement membrane proteins. In contrast to the expression of MMP19 in melanoma in which it is obviously upregulated first in Clark III, keratinocytes in the vicinity of melanoma cells seem to increase the expression of MMP19 also in Clark II stages. Thus, melanoma cells of initial stages may use the proteolytic potential of MMP19 from surrounding keratinocytes to invade the dermal compartment.

Unlikely from basal carcinoma and SCCs, in which MMP19 is downregulated during neoplastic dedifferentiation, ${ }^{46}$ MMP19 increases its expression in melanoma progression. The different expression patterns in tumours originating from keratinocytes and melanocytes raises questions as to how the expression of MMP19 is controlled in melanoma. Although speculative, we can draw several possibilities. There is probably a set of cytokines, growth factors, and extracellular matrix components that cooperate in the regulation of MMP19 in melanoma. Studies that tested the impact of several cytokines and growth factors are limited ${ }^{22,46}$ and, thus, it is difficult to figure out which of these factors might be responsible for regulation of MMP19. Nevertheless, 

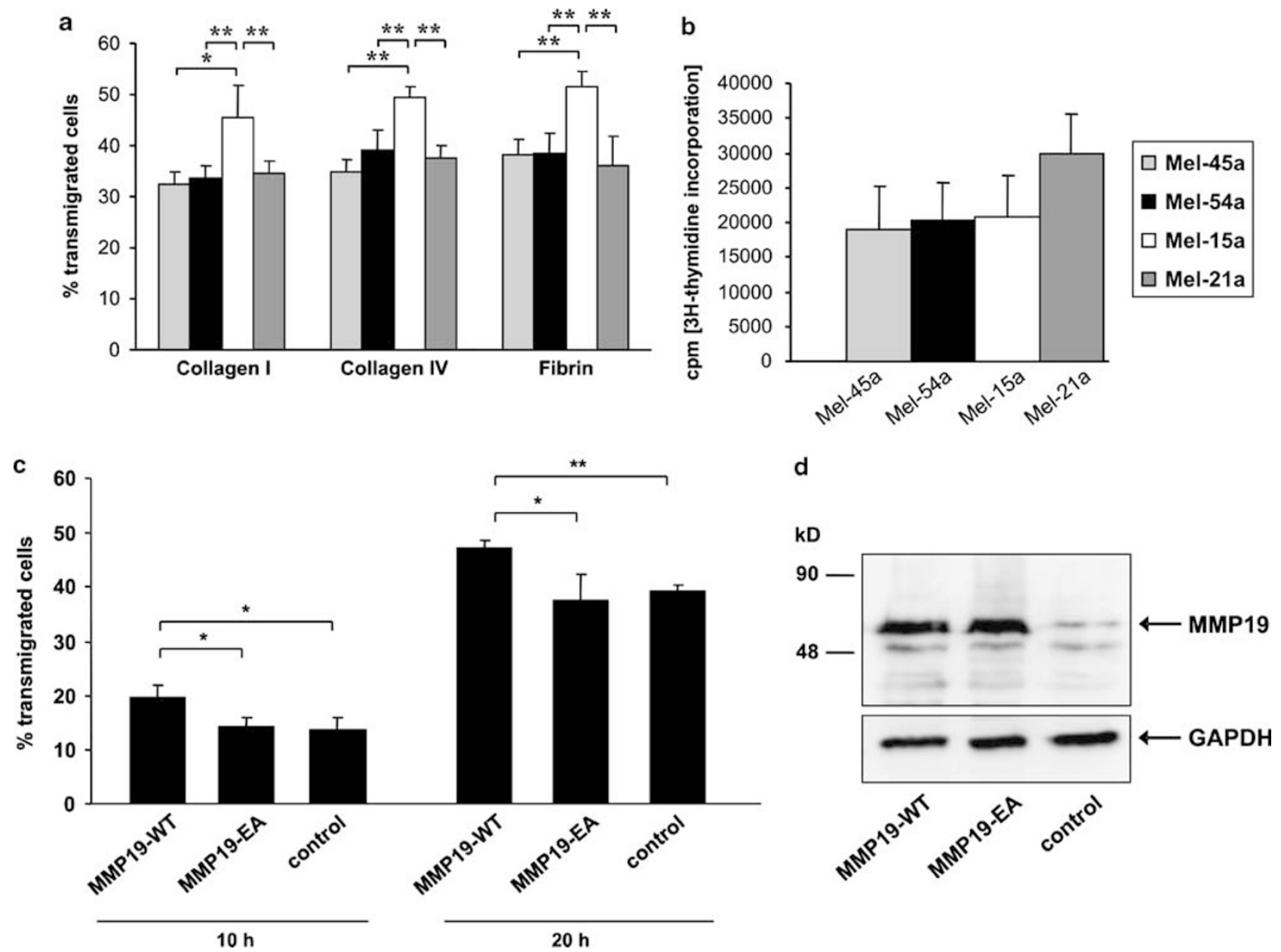

Figure 5 MMP19 in human melanoma cell lines Mel-45a, Mel-54a, Mel-15a, and Mel-21a. Transmigration through collagen I, collagen IV, and fibrin matrices (a). Melanoma cell lines exhibited similar proliferation measured by ${ }^{3} \mathrm{H}$-thymidine incorporation (b). MMP19 affects cell transmigration. Cell line Mel-21a was transiently transfected with MMP19-WT and MMP19-EA and tested for transmigratory capacity through collagen type IV matrix after 10 and $20 \mathrm{~h}$; the control, empty vector (c). Control of MMP19 expression in cell lysates was performed by western blotting $48 \mathrm{~h}$ after transfection (d). Significant differences are marked by asterisk $\left({ }^{*} P<0.05\right.$, ${ }^{* *} P<0.01$, Student's $t$-test).

high invasive melanoma cell lines have been reported as inducers of MMP1 in stromal fibroblasts by synthesis of interleukin- $1 \alpha,{ }^{13}$ whereas MMP9 was induced by TNF- $\alpha$ and interleukin- $1 \beta .{ }^{47}$ Both factors might also be of importance in the induction of MMP19 in melanoma. ${ }^{22,46}$ Extracellular matrix proteins are relevant regulators; type I and IV collagens that are also abundantly present in melanoma and its surroundings have been shown to upregulate MMP19 in keratinocytes. ${ }^{22}$

Another set of factors that might have a function is adhesion molecules and cell-cell interactions. E-cadherin, detected in early stages of melanoma, is lost during vertical growth and ${ }^{21,48,49}$ is replaced by $\mathrm{N}$-cadherin that is implicated in melanoma cell invasion, survival, and metastasis. ${ }^{48,49}$ MMP19 and E-cadherin are expressed in different epidermal compartments. Although E-cadherin is largely expressed in suprabasal layers, MMP19 is restricted to basal keratinocytes. ${ }^{32}$ As blocking anti-E-cadherin antibodies rescued expression of MMP19 in keratinocytes, ${ }^{32}$ similar regulatory E-cadherindependent mechanism might also be involved in releasing MMP19 expression after downregulation of E-cadherin during melanoma progression. This paradigm might function although both MMP19 and E-cadherin are expressed in Clark III melanomas. In this stage, E-cadherin is localized mostly to keratinocytes and less to melanoma cells, in which MMP19 is upregulated.

Another point in the regulation of MMP19 is its likely dependence on cell differentiation. MMP19 is lost during keratinocyte differentiation. ${ }^{22,32}$ As the development of melanoma is proposed to be either a stepwise process reminiscent of dedifferentiation or a development that originates from immature melanocytic cells, the regulation of MMP19 might parallel to the differentiation process in keratinocytes.

Upregulation of MMP19 in melanoma progression and metastasis exhibits an opposite pattern than other types of cancer. MMP19 is downregulated in 
mammary gland tumours during the progression towards an invasive phenotype and neoplastic dedifferentiation. ${ }^{23}$ Comparable disappearance could be found in basal and SCC. ${ }^{25,46}$ Similar pattern was seen only in oropharyngeal SCC in which MMP19 immunoreactivity positively correlated with tumour invasiveness. ${ }^{50}$ The exact contribution of MMP19 in melanoma progression remains to be investigated. However, the initial experiments performed indicated that MMP19 expression correlated with higher migratory capacity and low adhesion of melanoma cells and its ectopic expression increased migratory potential through type IV collagen.

In summary, we found that MMP19 expression was upregulated in the vertical growth phase and in metastases, suggesting participation of MMP19 in melanoma development and MMP19 as a candidate marker for identifying vertical growth phase melanoma and metastatic melanomas.

\section{Acknowledgements}

We gratefully thank E. Schulz for excellent technical assistance. Financial support was given to RS and EP by the DFG (SFB 617), by AV0Z50520514, Academy of Sciences of the Czech Republic to RS, and KR by the DFG (SFB 617) and the Centre of Excellence 'Inflammation at Interfaces'.

\section{Disclosure/conflict of interest}

The authors declare no conflict of interest.

\section{References}

1 Curran S, Murray GI. Matrix metalloproteinases in tumour invasion and metastasis. J Pathol 1999;189: 300-308.

2 Hofmann UB, Houben R, Brocker EB, et al. Role of matrix metalloproteinases in melanoma cell invasion. Biochimie 2005;87:307-314.

3 Hofmann UB, Westphal JR, Van Muijen GN, et al. Matrix metalloproteinases in human melanoma. J Invest Dermatol 2000;115:337-344.

4 Herlyn M, Clark WH, Rodeck U, et al. Biology of tumor progression in human melanocytes. Lab Invest 1987; 56:461-474.

5 Clark Jr WH, From L, Bernardino EA, et al. The histogenesis and biologic behavior of primary human malignant melanomas of the skin. Cancer Res 1969;29: 705-727.

6 Breslow A. Thickness, cross-sectional areas and depth of invasion in the prognosis of cutaneous melanoma. Ann Surg 1970;172:902-908.

7 Stetler-Stevenson WG, Liotta LA, Kleiner DE, et al. Extracellular matrix 6: role of matrix metalloproteinases in tumor invasion and metastasis. FASEB J 1993;7:1434-1441.

8 Nagase H, Woessner Jr JF. Matrix metalloproteinases. J Biol Chem 1999;274:21491-21494.
9 Airola K, Karonen T, Vaalamo M, et al. Expression of collagenases- 1 and -3 and their inhibitors TIMP-1 and -3 correlates with the level of invasion in malignant melanomas. Br J Cancer 1999;80:733-743.

10 Hofmann UB, Westphal JR, Waas ET, et al. Matrix metalloproteinases in human melanoma cell lines and xenografts: increased expression of activated matrix metalloproteinase-2 (MMP-2) correlates with melanoma progression. Br J Cancer 1999;81: 774-782.

11 Huntington JT, Shields JM, Der CJ, et al. Overexpression of collagenase 1 (MMP-1) is mediated by the ERK pathway in invasive melanoma cells: role of BRAF mutation and fibroblast growth factor signaling. J Biol Chem 2004;279:33168-33176.

12 Iida J, McCarthy JB. Expression of collagenase-1 (MMP-1) promotes melanoma growth through the generation of active transforming growth factor-beta. Melanoma Res 2007;17:205-213.

13 Loffek S, Zigrino P, Angel P, et al. High invasive melanoma cells induce matrix metalloproteinase-1 synthesis in fibroblasts by interleukin-1alpha and basic fibroblast growth factor-mediated mechanisms. J Invest Dermatol 2005;124:638-643.

14 Hofmann UB, Westphal JR, Zendman AJ, et al. Expression and activation of matrix metalloproteinase-2 (MMP-2) and its co-localization with membrane-type 1 matrix metalloproteinase (MT1-MMP) correlate with melanoma progression. J Pathol 2000; 191:245-256.

15 Simonetti O, Lucarini G, Brancorsini D, et al. Immunohistochemical expression of vascular endothelial growth factor, matrix metalloproteinase 2, and matrix metalloproteinase 9 in cutaneous melanocytic lesions. Cancer 2002;95:1963-1970.

16 Woolley DE, Grafton CA. Collagenase immunolocalization studies of cutaneous secondary melanomas. Br J Cancer 1980;42:260-265.

17 Corte MD, Gonzalez LO, Corte MG, et al. Collagenase-3 (MMP-13) expression in cutaneous malignant melanoma. Int J Biol Markers 2005;20:242-248.

18 Kuivanen T, Ahokas K, Virolainen S, et al. MMP-21 is upregulated at early stages of melanoma progression but disappears with more aggressive phenotype. Virchows Arch 2005;447:954-960.

19 van den Oord JJ, Paemen L, Opdenakker G, et al. Expression of gelatinase $\mathrm{B}$ and the extracellular matrix metalloproteinase inducer EMMPRIN in benign and malignant pigment cell lesions of the skin. Am J Pathol 1997;151:665-670.

20 Gruss C, Herlyn M. Role of cadherins and matrixins in melanoma. Curr Opin Oncol 2001;13:117-123.

21 Sanders DS, Blessing K, Hassan GA, et al. Alterations in cadherin and catenin expression during the biological progression of melanocytic tumours. Mol Pathol 1999;52:151-157.

22 Sadowski T, Dietrich S, Muller M, et al. Matrix metalloproteinase-19 expression in normal and diseased skin: dysregulation by epidermal proliferation. J Invest Dermatol 2003;121:989-996.

23 Djonov V, Hogger K, Sedlacek R, et al. MMP-19: cellular localization of a novel metalloproteinase within normal breast tissue and mammary gland tumours. J Pathol 2001;195:147-155.

24 Impola U, Jeskanen L, Ravanti L, et al. Expression of matrix metalloproteinase (MMP)-7 and MMP-13 and loss of MMP-19 and p16 are associated with malignant 
progression in chronic wounds. $\mathrm{Br} \mathrm{J}$ Dermatol 2005;152:720-726.

25 Sadowski T, Dietrich S, Koschinsky F, et al. Matrix metalloproteinase 19 processes the laminin 5 gamma 2 chain and induces epithelial cell migration. Cell Mol Life Sci 2005;62:870-880.

26 Mauch S, Kolb C, Kolb B, et al. Matrix metalloproteinase-19 is expressed in myeloid cells in an adhesiondependent manner and associates with the cell surface. J Immunol 2002;168:1244-1251.

27 van Horssen J, Vos CM, Admiraal L, et al. Matrix metalloproteinase-19 is highly expressed in active multiple sclerosis lesions. Neuropathol Appl Neurobiol 2006;32:585-593.

28 Behera AK, Hildebrand E, Scagliotti J, et al. Induction of host matrix metalloproteinases by Borrelia burgdorferi differs in human and murine lyme arthritis. Infect Immun 2005;73:126-134.

29 Titz B, Dietrich S, Sadowski T, et al. Activity of MMP19 inhibits capillary-like formation due to processing of nidogen-1. Cell Mol Life Sci 2004;61:1826-1833.

30 Stracke JO, Fosang AJ, Last K, et al. Matrix metalloproteinases 19 and 20 cleave aggrecan and cartilage oligomeric matrix protein (COMP). FEBS Lett 2000; 478:52-56.

31 Stracke JO, Hutton M, Stewart M, et al. Biochemical characterization of the catalytic domain of human matrix metalloproteinase 19. Evidence for a role as a potent basement membrane degrading enzyme. J Biol Chem 2000;275:14809-14816.

32 Sadowski T, Dietrich S, Koschinsky F, et al. Matrix metalloproteinase 19 regulates insulin-like growth factor-mediated proliferation, migration, and adhesion in human keratinocytes through proteolysis of insulinlike growth factor binding protein-3. Mol Biol Cell 2003;14:4569-4580.

33 Kolb C, Mauch S, Krawinkel U, et al. Matrix metalloproteinase-19 in capillary endothelial cells: expression in acutely, but not in chronically, inflamed synovium. Exp Cell Res 1999;250:122-130.

34 Hussein MR. Analysis of Bcl-2 protein expression in choroidal melanomas. J Clin Pathol 2005;58:486-489.

35 Sun Y, Song M, Stevanovic S, et al. Identification of a new HLA-A ${ }^{*}$ )0201-restricted T-cell epitope from the tyrosinase-related protein 2 (TRP2) melanoma antigen. Int J Cancer 2000;87:399-404.

36 Beck IM, Muller M, Mentlein R, et al. Matrix metalloproteinase-19 expression in keratinocytes is repressed by transcription factors Tst-1 and Skn-1a: implications for keratinocyte differentiation. J Invest Dermatol 2007;127:1107-1114.

37 MacDougall JR, Bani MR, Lin Y, et al. The 92-kDa gelatinase B is expressed by advanced stage melanoma cells: suppression by somatic cell hybridization with early stage melanoma cells. Cancer Res 1995;55: 4174-4181.
38 Kurschat P, Wickenhauser C, Groth W, et al. Identification of activated matrix metalloproteinase-2 (MMP-2) as the main gelatinolytic enzyme in malignant melanoma by in situ zymography. J Pathol 2002;197: 179-187.

39 Durko M, Navab R, Shibata HR, et al. Suppression of basement membrane type IV collagen degradation and cell invasion in human melanoma cells expressing an antisense RNA for MMP-1. Biochim Biophys Acta 1997;1356:271-280.

40 Montgomery AM, De Clerck YA, Langley KE, et al. Melanoma-mediated dissolution of extracellular matrix: contribution of urokinase-dependent and metalloproteinase-dependent proteolytic pathways. Cancer Res 1993;53:693-700.

41 Mueller BM. Different roles for plasminogen activators and metalloproteinases in melanoma metastasis. Curr Top Microbiol Immunol 1996;213:65-80.

42 MacDougall JR, Bani MR, Lin Y, et al. 'Proteolytic switching' opposite patterns of regulation of gelatinase $\mathrm{B}$ and its inhibitor TIMP-1 during human melanoma progression and consequences of gelatinase $\mathrm{B}$ overexpression. Br J Cancer 1999;80:504-512.

43 Vaisanen A, Tuominen $\mathrm{H}$, Kallioinen M, et al. Matrix metalloproteinase-2 (72 kD type IV collagenase) expression occurs in the early stage of human melanocytic tumour progression and may have prognostic value. J Pathol 1996;180:283-289.

44 Vaisanen A, Kallioinen M, Taskinen PJ, et al. Prognostic value of MMP-2 immunoreactive protein (72 kD type IV collagenase) in primary skin melanoma. J Pathol 1998;186:51-58.

45 Walker RA, Woolley DE. Immunolocalisation studies of matrix metalloproteinases-1, -2 and -3 in human melanoma. Virchows Arch 1999;435:574-579.

46 Impola U, Toriseva M, Suomela S, et al. Matrix metalloproteinase-19 is expressed by proliferating epithelium but disappears with neoplastic dedifferentiation. Int J Cancer 2003;103:709-716.

47 Shellman YG, Makela M, Norris DA. Induction of secreted matrix metalloproteinase-9 activity in human melanoma cells by extracellular matrix proteins and cytokines. Melanoma Res 2006;16:207-211.

$48 \mathrm{Hsu} \mathrm{M}$, Andl T, Li G, et al. Cadherin repertoire determines partner-specific gap junctional communication during melanoma progression. J Cell Sci 2000;113:1535-1542.

49 Krengel S, Groteluschen F, Bartsch S, et al. Cadherin expression pattern in melanocytic tumors more likely depends on the melanocyte environment than on tumor cell progression. J Cutan Pathol 2004;31:1-7.

50 Velinov N, Aebersold D, Haeni N, et al. Matrix metalloproteinase-19 is a predictive marker for tumor invasiveness in patients with oropharyngeal squamous cell carcinoma. Int J Biol Markers 2007;22: 265-273. 